\title{
Improvement of ship operational safety as a result of the application of virtual reality engine room simulators
}

\author{
R. Cwilewicz \& L. Tomczak \\ Marine Propulsion Plant Department, Gdynia Maritime University, \\ Poland
}

\begin{abstract}
Engine room simulators have been used in maritime academies as a valuable asset to the educational process for more than 30 years. The present development of personal computers, modern processors and graphical cards allows for the easy application of 3D simulation techniques and for this reason manufacturers of engine room simulators have begun to apply tri-dimensional graphical system layout presentation. Their aim is to provide machinery simulation as close as possible to reality. As a result, trainees are far better prepared to deal with the real life operation of machinery, thus considerably increasing the standards of ship operational safety. Additionally, in simulation conditions, it is possible to acquaint trainees with emergency situations that may not be performed in real life conditions due to safety exigencies.

Experiences in the application of virtual visualisation, and the benefits and advantages of the use of engine room simulators in the educational process of engine room officers are equally presented in this paper. This paper describes an example of the application of new 3D simulation techniques in an engine room simulator based on a modern computer controlled engine room with a medium speed main engine, applied on a container ship.

The simulator described in this paper provides for a new approach to navigation through the different system elements, allowing for quick and easy access to basic engine room operation (valve opening/closing, setting position of switches, push-buttons etc.). This has been possible due to the application of state-of-the art $3 \mathrm{D}$ visualisation with zoom techniques.

Keywords: 3D computer simulation, marine engine room simulators.
\end{abstract}




\section{Introduction}

Nowadays, tremendous changes are taking place in computing, information technology and simulation. Maritime education and training is not isolated from such changes and ought to benefit from these tendencies, especially taking into account the possible improvements for ship operational safety. Marine engine simulators allow for the operation of emergency situations that are not permissible under normal exploitation conditions due to safety limitations. Simulator software also includes assessment features that enable the objective review of trainees' acquired capacities. During a simulator exercise the instructor is able to apply various exercise set-ups (initial conditions) and scenarios that include different fault finding tasks.

For this reason, engine room simulators are being used more and more used in maritime academies as a valuable asset for educational process [1]. The application of engine room simulators is also recommended by the STCW 95 IMO Convention [2].

It is worthwhile mentioning that marine engine room simulators also have some basic disadvantages. Namely, they include lots of simplifications, abbreviations and schematic presentation of machinery systems as a result of the fact that they are presented only in a $2 \mathrm{D}$ visualization. Hence, the trainee with a perfect knowledge of simulator operation can experience serious problems with real ship power plant operation, because the graphical presentation and operating procedures of the simulator are distinct from the reality.

For this reason, manufacturers of engine room simulators have begun to apply 3D graphical system layout presentation in order to provide a machinery configuration as close as possible to reality.

The main problem in the creation of $3 \mathrm{D}$ simulators is to provide for proper navigation through the system's elements [4-6]. The engine room is a complex, multi level and complicated set of sub-systems, equipment and machinery and this is a new challenge for entities creating such kinds of simulators.

It is also necessary to allow for quick and easy access to basic engine room operation (valve opening/closing, setting position of switches, push-buttons etc.). It is possible to achieve this feature by applying zoom techniques for selected elements of the system. Users of 3D simulators should also be able to observe the system's elements from pre-selected specific parts of the engine room.

Based on the author's experiences with the application of different types of simulators, a better solution consists in navigation by mouse cursor and zooming facilities.

The application of new 3D simulation techniques in marine engineering education shall be analysed on the examples of a full mission, hardware type engine room simulator with medium speed main engine.

A new technique for navigation through the system elements has been applied in this virtual reality simulator, providing a solution to the main problem in the creation of 3D visualisation. The latest development also includes a combination of 3D and 2D diagram presentation, which enables one to follow how a certain device really functions and gives a complete picture of its structure. The 
presented solutions have considerably improved the level of simulator fidelity in relation to real machinery. In consequence, it was possible to eliminate the disadvantages of the engine room simulator with typical 2D presentation consisting of a schematic and simplified presentation of machinery systems.

The application of virtual simulation in teaching the operation of complex marine machinery leads to a better understanding of the functioning principles of both the equipment and the systems in comparison with traditional educational methods. As a result, trainees are far better prepared to deal with the real life operation of machinery, thus considerably increasing standards of ship operational safety. It has been observed during many years of application of engine room simulators in Gdynia Maritime University that trainees have a very different approach to exercises conducted with hardware types of simulator in comparison with training conducted only with software. The trainee's attitude to exercises conducted with software only does not differ from the approach adopted to computer games and the trainee often does not consider these as serious experiences that reflect reality. If the exercise is conducted with the application of hardware, the trainees have the impression of conducting real life operations and in consequence, their aptitude to perform such operations in reality increases. On the other hand, software, due to its features enables the trainee to repeat in an unlimited number of times the required operations, thus achieving the necessary preparedness level.

The engine room simulator based on the medium speed engine room simulator is one of the first simulators that use the hardware type of consoles combined with $3 \mathrm{D}$ visualization.

This simulator especially enhances the operational procedures related to emergency situations, such as electrical black-down, emergency manual operation of the main engine with the propulsion system, as well as auxiliary machinery in case of remote control failure. As has been said before, these procedures may not be trained for in real life conditions due to safety constraints. From a didactic point of view the best solution is to combine the hardware version of the engine room simulator with $3 \mathrm{D}$ visualization. Such a combination considerably improves the safe operation of the marine engine room, as the crew members have previously been trained in relation to various fault scenarios.

\section{MED3 medium speed engine room simulator description}

It is well known that the level of theoretical and practical knowledge possessed by engine room officers while operating engines and auxiliary equipment is directly linked to accident prevention on board.

The basic role of this simulator is the familiarization with different operational modes. The more familiar the trainee is with the equipment the faster and more effective are his reactions in a state of emergency [3].

The MED3DH is a full mission, hardware type medium speed engine simulator and has been based on very modern solutions, presently being used in medium-sized, computer controlled, engine rooms (one four-stroke type main engine with reduction gear and controllable pitch propeller). 
This kind of computer controlled engine room is a typical configuration applied on modern container ships.

The MED3DH virtual reality simulator provides a new approach to navigation through the different system elements, allowing for quick and easy access to basic engine room operation (valve opening/closing, setting position of switches, push-buttons etc.). This has been possible due the application of state-of-the-art 3D visualisation with zoom techniques. The latest development also includes a combination of 3D and 2D diagram presentation, which enables one to follow how a certain device really functions and gives a complete picture of its structure. The presented solutions have considerably improved the level of simulator fidelity in relation to real machinery. In consequence, it was possible to eliminate the disadvantages of the engine room simulator with typical 2D presentation consisting of a schematic and simplified presentation of machinery systems.

This simulator is designated for training students of maritime academies as well as for different types of marine vocational training centres. The simulator has universal features and may be used both for training merchant and navy fleet crew.

The main purpose of the MED3DH simulator is the practical preparation of the trainee for engine room operation, and more particularly:

- Familiarization with the basic engine room installation (compressed air system, fresh and sea water cooling system, lubrication, fuel oil system etc.), especially taking into account training on the basis of a modern, computer controlled engine room;

- Acknowledgment of main engine and auxiliary equipment exploitation procedures;

- $\quad$ Propulsion system manoeuvring (main engine - reduction gear - CPP).

The software allows for the simulation of opening/closing of basic valves and auxiliary equipment operation in the engine room. The software also generates the main engine room's sound.

The simulator has been developed in compliance with:

- STCW Code: Section A-1/12 and Section B-1/12.

- ISM Code: Section 6 and Section 8.

The MED3DH simulator has been equipped with hardware consoles and panels that enable the operation and control of the main engines, gear, CPP, auxiliary machinery and electrical system.

The MED3DH simulator consists of three main elements:

- Engine Control Room ECR with main engine control console and main electric switchboard,

- Engine Room with two PC projectors and control console for 3D visualization combined with diagram presentation,

- Instructor's Room.

The main engine starting and stopping procedure is effectuated on hardware type control consoles and Electric Switchboard panels.

A general view of the MED3D simulator rooms has been presented in Figs. 1-3. 


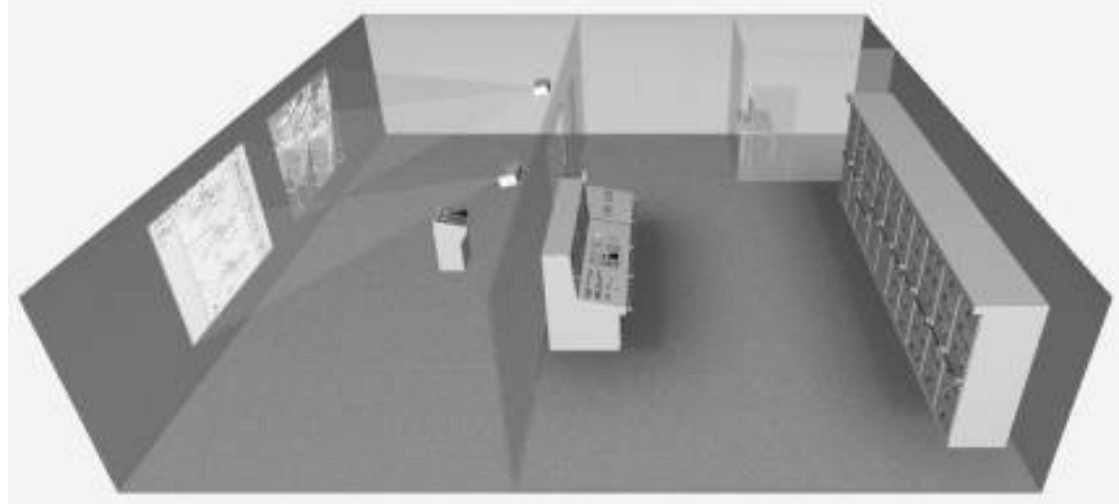

Figure 1: General view of the full mission engine room simulator MED3DH - hardware version.

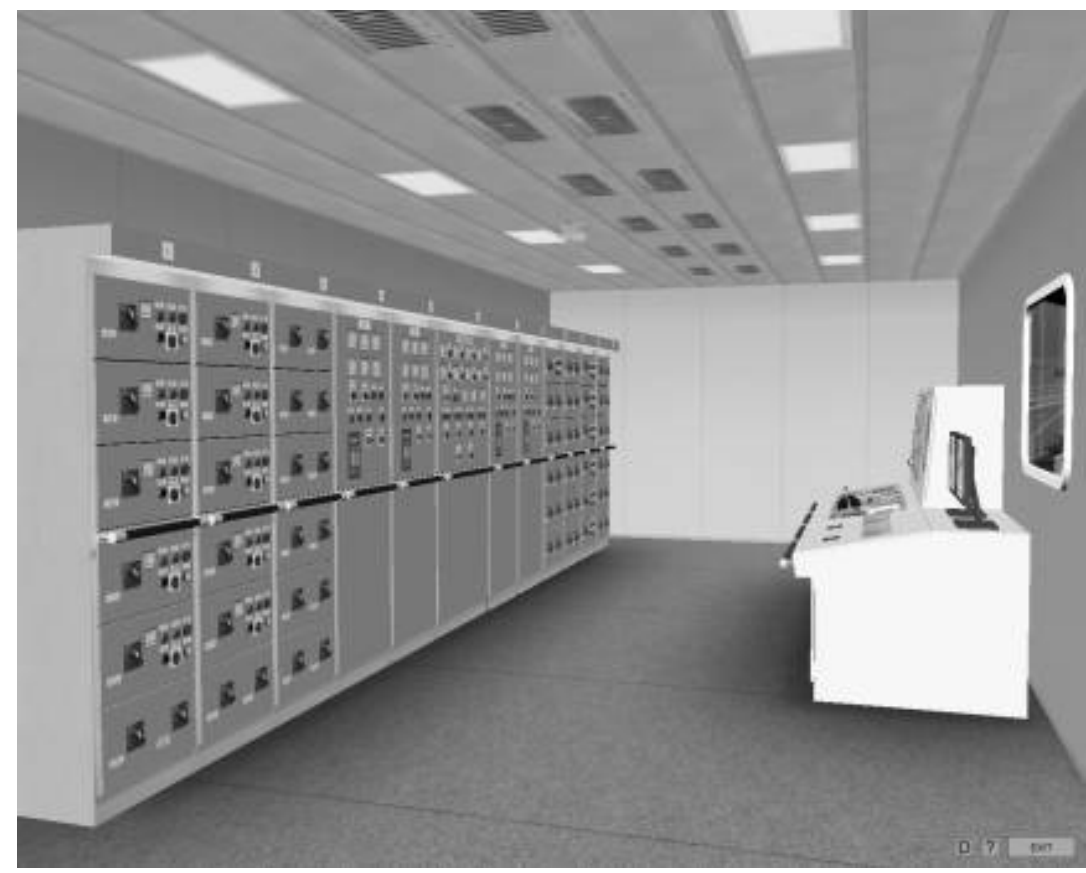

Figure 2: General view of the MED3D control room - hardware version. 


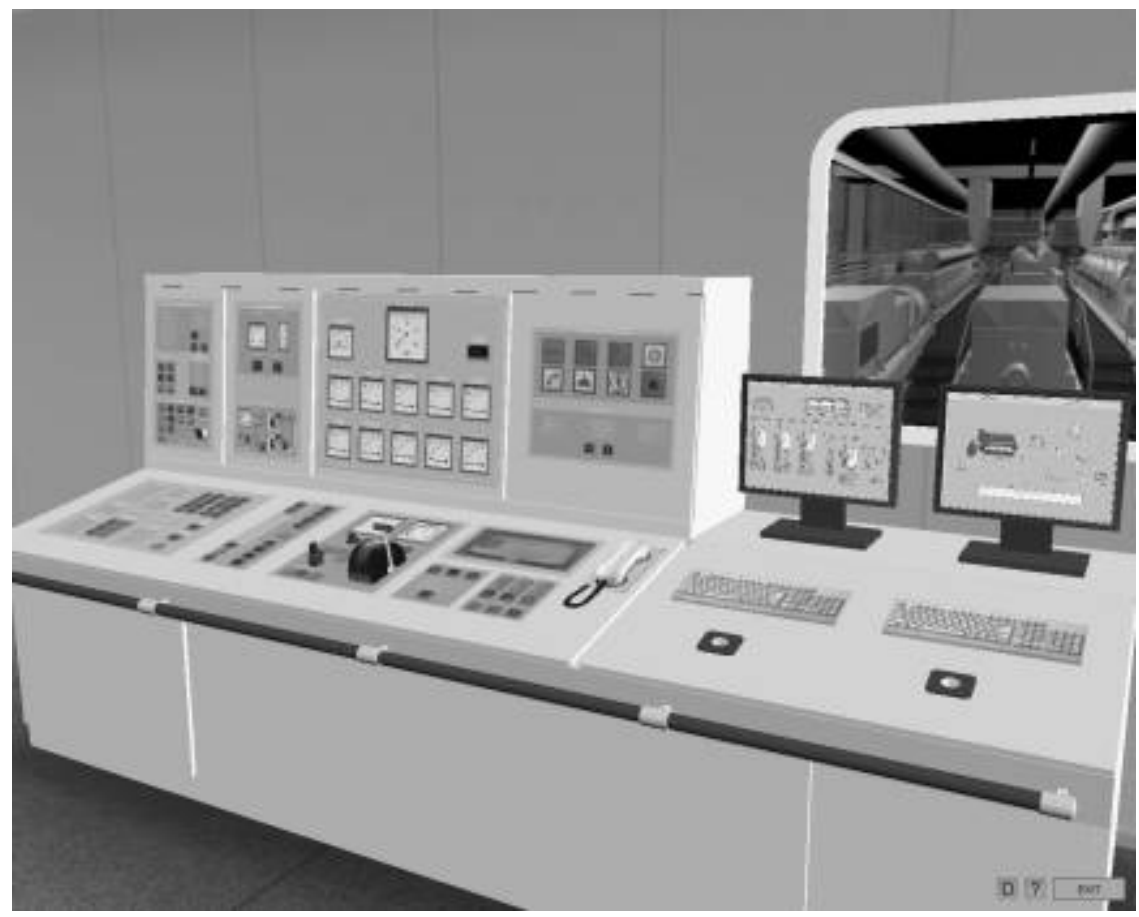

Figure 3: $\quad$ MED3D main engine control hardware console.

The basic operation with auxiliary sub-systems, identical to a real modern ship, is performed by a "mouse clicking" operation on the PC monitor screen (Fig. 4). On the PC screen diagrams related to specific installations such as fuel oil, compressed air system, cooling system, lubricating system, steam system, etc. are presented. For security reasons and in the same way as in a real engine room, the Engine Control Room is equipped with two identical PCs with monitors.

In the case of one PC failure, the second one takes over the systems' control automatically. On the monitor screen by "mouse clicking" it is possible to start/stop the pumps, compressors and open/close the valves which are remote controlled.

All operating procedures that need to be performed in engine rooms (outside of the Engine Control Room) are effectuated in a separate room equipped with two projector screens (Fig. 1).

One screen presents engine room elements in $3 \mathrm{D}$ visualization and another one presents in the form of a diagram related to specific and selected compartments or systems of the engine rooms, for example auxiliary generator room, emergency generator room, compressed system room etc. The selection of engine room elements that are important from the point of view of exploitation is performed by zoom techniques. Fig. 5 presents an example of the steam system diagram and in Figs. 6 and $73 \mathrm{D}$ visualization of this part of engine room is shown. 


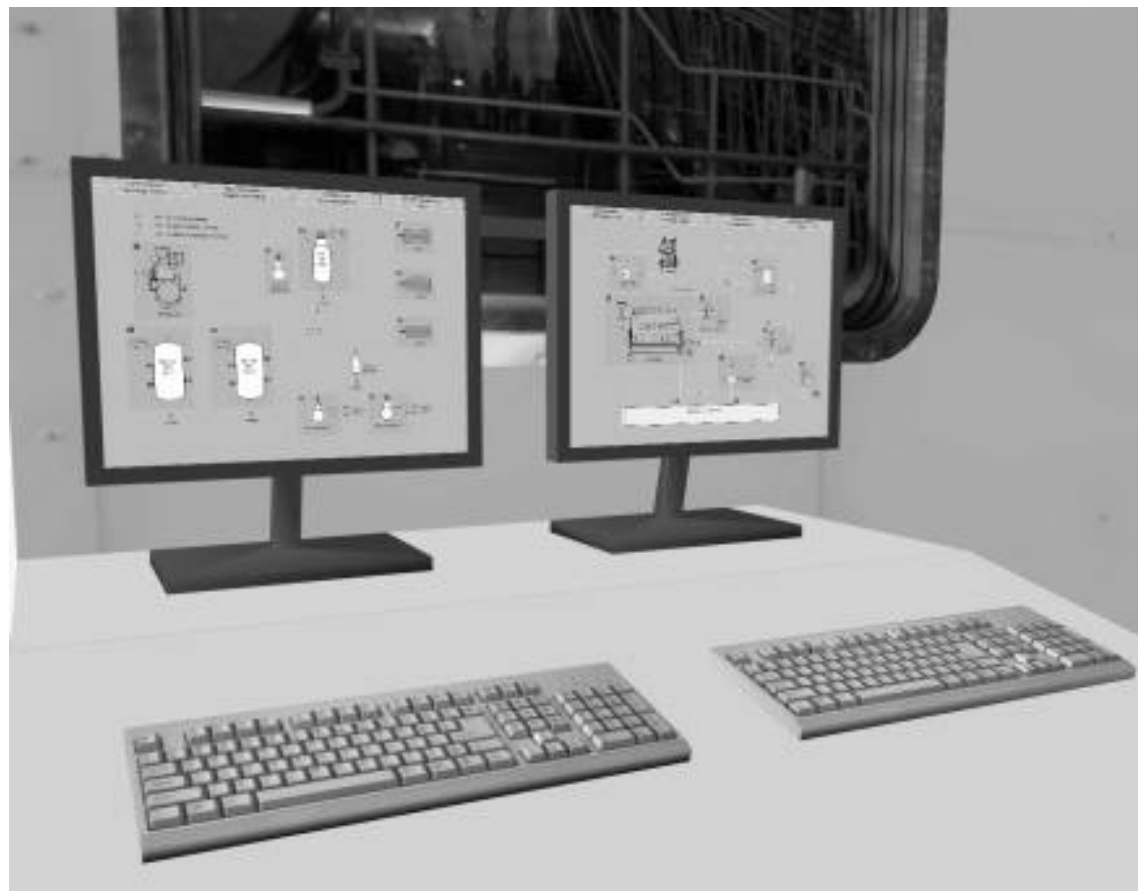

Figure 4: $\quad$ PC monitors in the engine control room.

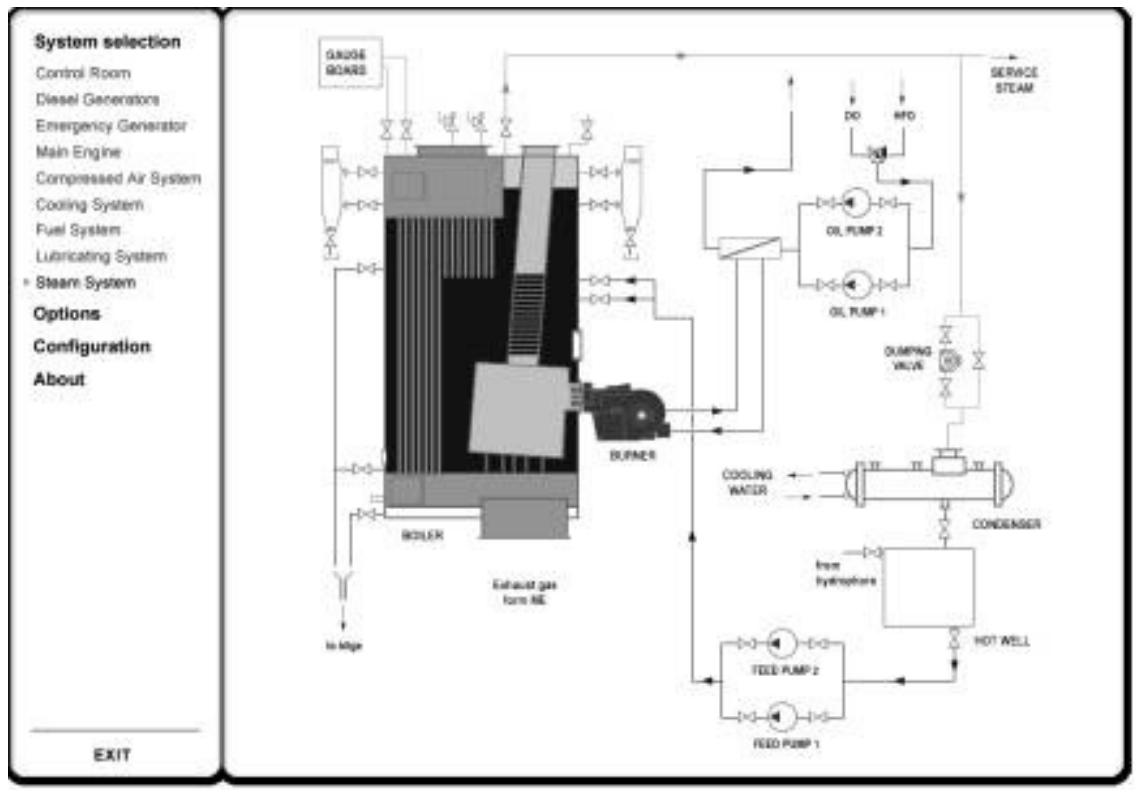

Figure 5: Steam system diagram. 


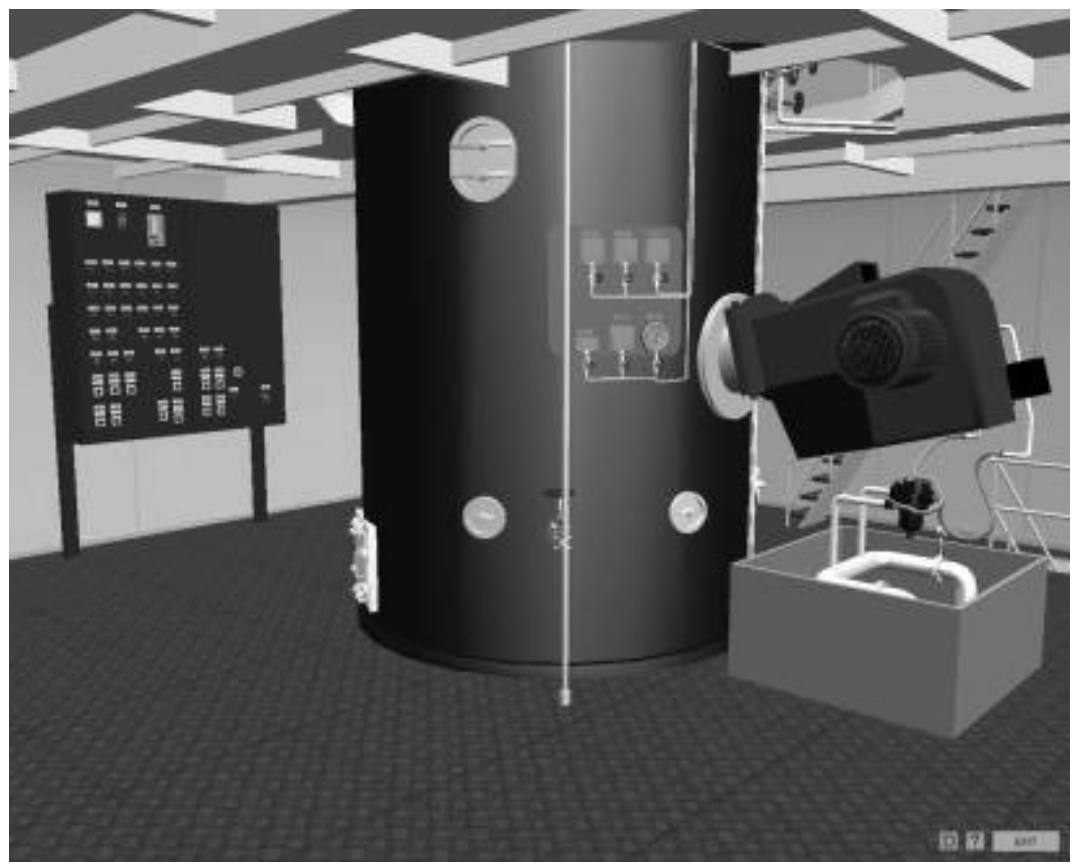

Figure 6: $\quad$ Steam boiler - 3D visualization (lower part).

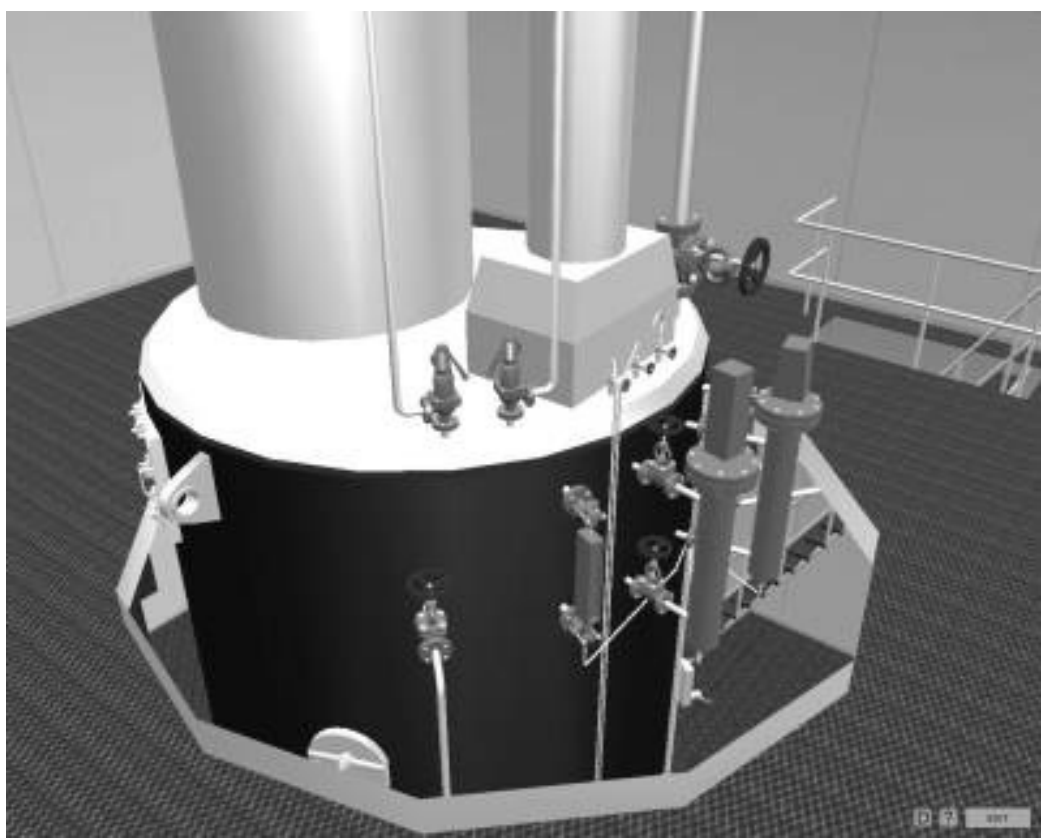

Figure 7: $\quad$ Steam boiler - 3D visualization (upper part).

WIT Transactions on Information and Communication, Vol 39, (C) 2008 WIT Press www.witpress.com, ISSN 1743-3517 (on-line) 


\section{Conclusion}

As has been mentioned above, a simulator that combines hardware with $3 \mathrm{D}$ visualization considerably improves the operational safety of marine engines as it enables the trainee to achieve a high level of emergency preparedness. The didactic purposes are achieved through training involving various fault scenarios and the simulator also enables proper navigation through its elements. The new concepts of view selection, zooming features of elements and operation by cursor and mouse clicking, as applied in the Emergency Power Plant simulator described in this paper, appear to be very effective and easily adaptable by trainees in practice.

Due to the specificity of operating marine equipment in real life conditions, the didactic goals in marine education are directly linked with achieving preparedness for emergency situations. Such preparedness may only be achieved if the trainee is familiar with both the equipment and its operating modes, including emergency situations. If the trainee has been trained only on simulators that are far from the real machinery environment, his state of preparation for emergencies on board is not satisfactory and safety is put at risk.

$3 \mathrm{D}$ visualisation reduces the gap between operating marine machinery in simulation conditions and in real life. In the near future, this type of 3D solution should be applied more and more often in engine room simulator design. The presented simulators are related to marine machinery, but the concept of composition and navigation through the system elements can be easily applied for the purposes of any type of technical equipment and shall contribute in a similar manner to hazard prevention.

To summarize, the application of engine room simulators with 3D visualization improves the operational safety of marine equipment on board as it reduces the level of human error in the operation and maintenance of these devices.

\section{References}

[1] R.Cwilewicz, L. Tomczak, Z.J. Pudlowski, Effective application of engine room simulators in marine engineering education, Proc. $3^{\text {rd }}$ Global Conference on Engineering Education, Glasgow, Scotland, United Kingdom, 2002, pp. 316-318.

[2] STCW - Standards of Training, Certification and Watchkeeping for Seafarers 78/95 Convention International Maritime Organization, London, 1996

[3] R. Cwilewicz, L. Tomczak, The role of computer simulation programs for marine engineers in hazard prevention by reducing the risk of human error in the operation of marine machinery, Proc. $4^{\text {th }}$ International Conference on Computer Simulation in Risk Analysis and Hazard Mitigation - Risk Analysis IV, 2004, pp. 245-249 
[4] L. Tomczak, Practical aspects of 3D graphical applications in marine Engineering Education, Global Journal of Engineering Education, 9(2), 2005, pp.137-142.

[5] L. Tomczak, Application of 3D visualization in marine engine room simulators, Proc. $7^{\text {th }}$ International Conference on Engine Room Simulators (ICERS7), Portoroz, Slovenia, 2005, pp. 232-240.

[6] L. Tomczak, The latest developments of $3 \mathrm{D}$ visualization in marine engine room simulators, Proc. $8^{\text {th }}$ International Conference on Engine Room Simulators (ICERS8), Manila, Philippines, 2008, pp. 40-42. 